\title{
Evaluation of Turkish wild Emmers (Triticum dicoccoides Koern.) and wheat varieties for resistance to the root lesion nematodes (Pratylenchus thornei and Pratylenchus neglectus) ${ }^{1}$
}

\author{
Türkiye'de bulunan yabani Emmer buğdayları (Triticum dicoccoides Koern.) ve buğday \\ çeşitlerinin Kök lezyon nematodları (Pratylenchus thornei and P. neglectus)'na karşı \\ dayanıklılıklarının belirlenmesi

\section{Halil TOKTAY ${ }^{2^{*}}$ Mustafa IMREN ${ }^{3}$ İbrahim Halil ELEKCiOĞLU ${ }^{4}$ Abdelfattah A. DABABAT ${ }^{5}$ Summary}

Root-lesion nematodes (RLN; Pratylenchus thornei and Pratylenchus neglectus) are serious plant parasitic nematodes that attack wheat roots and cause significant losses in grain quality and quantity worldwide. They are widely distributed in Turkish wheat growing areas and can be only controlled by growing resistant cultivars and nonhost crops. So far, there is no commercial wheat cultivar identified as completely resistant to the RLN is available. The objective of this research was to evaluate some Turkish wild Emmers (Triticum dicoccoides) and national spring wheat varieties for resistant to $P$. thornei and $P$. neglectus. In the experiments, 32 wild Emmers and 42 wheat cultivars were inoculated with $P$. thornei or $P$. neglectus individuals and grown under controlled conditions in a growth room for 9 weeks. The results showed that 25 and 35 wheat varieties were moderately resistant to $P$. thornei and $P$. neglectus, respectively. In total, 17 lines exhibiting multiple resistance reactions to both nematodes were in the same group with the check lines $(P<0.05)$. The identified resistant wheat accessions showed improved levels of RLN resistance over current parents and could serve as an excellent genetic pool to be used in an efficient Turkish wheatbreeding programme.

Keywords: Wheat, wild emmer, root lesion nematodes, Pratylenchus spp., resistance

\section{Özet}

Kök lezyon nematodları (RLN; Pratylenchus thornei ve $P$. neglectus) buğday köklerine saldırarak, tahıl kalitesi ve miktarında küresel olarak önemli kayıplara neden olan önemli bir bitki paraziti nematod gurubudur. Türkiye'de buğday ekiliş alanlarında yaygın olarak bulunan bu nematodlarla ancak dayanıklı çeşitler ve rotasyonla mücadele etmek mümkündür. Maalesef bugüne kadar bu nematod gurubuna karşı tamamen dayanıklı olarak tanımlanan hiçbir ticari buğday çeşidi bulunamamıştır. Bu nedenle, buğday ıslah programlarında yüksek verimli ve kaliteli buğday çeşitlerine orta derecede (kısmi) dayanıklı ebeveynler kullanılarak dayanıklılık entegre edilmeye çalışıımıştır. Bu araştırmanın amacı bazı Türk yabani Emmer'lerinin (Triticum dicoccoides) ve bazı ulusal yazlık buğday çeşitlerinin $P$. thornei ve $P$. neglectus'a karşı dayanıklılık durumlarını ortaya koymaktır. Bu amaçla her iki nematoda karşı yapılan iki farklı denemede, 32 yabani Emmer buğdayı ve 42 buğday çeşidinin, $P$. thornei veya $P$. neglectus bireylerine karşı dayanıklıkları 9 hafta boyunca kontrollü koşullar altında testlenmiştir. Sonuç olarak, $P$. thornei ve $P$. neglectus'a karşı sırasıyla 25 ve 35 buğday çeşidinin orta derecede dayanıklılık reaksiyonu sağladığı belirlenmiştir. Toplamda 17 çeşit her iki nematoda karşı dayanıklılık göstermiştir $(p<0.05)$. Elde edilen verilere göre, tespit edilen dayanıklı buğday çeşitlerinin mevcut hatlardan daha dayanıklı olduğu belirlenmiş ve bu hatların Türkiye'deki ıslah programlarında mükemmel bir şekilde kullanılabileceği ortaya konmuştur.

Anahtar Sözcükler: Buğday, yabani emmer, kök yara nematodları, Pratylenchus spp., dayanıklılı

\footnotetext{
${ }^{1}$ Bu çalışma Tübitak tarafından desteklenen 1120565 nolu projenin bir kısmıdır.

${ }^{2}$ Niğde Üniversitesi Ayhan Şahenk Tarım Bilimleri ve Teknolojileri Fakültesi, Bitkisel Üretim ve Teknolojileri Bölümü, Merkez Yerleşke, 51240, Niğde, Türkiye

${ }^{3}$ Abant İzzet Baysal Üniversitesi Ziraat ve Doğa Bilimleri Fakültesi, Bitki Koruma Bölümü, 14280 Gölköy, Bolu, Türkiye

${ }_{5}^{4}$ Çukurova Üniversitesi, Ziraat Fakültesi, Bitki Koruma Bölümü, 01360 Balcalı, Adana, Türkiye

${ }^{5}$ CIMMYT (International Maize and Wheat Improvement Centre) P.K. 39 06511, Emek, Ankara, Turkey

* Sorumlu yazar (Corresponding author) e-mail: h.toktay@nigde.edu.tr

Alınış (Received): 09.10.2014 Kabul ediliş (Accepted): 11.06.2015 


\section{Introduction}

Turkey is considered 10th wheat (Triticum aestivum) producing country in the world with 20 million tonnes in 9 million hectares per year (TUIK, 2013). Plant parasitic nematodes are important biotic agents that cause substantial yield loss in semiarid wheat growing regions. Root-lesion nematodes (RLN; Pratylenchus spp.) damage plants mechanically and chemically, reduce plant vigour and can cause root lesions that predispose plants to infection by root-infecting fungi, which may lead to the formation of a disease complex. There are eight species of RLN that have been recorded for small grains (Rivoal \& Cook, 1993). Of these four species (Pratylenchus thornei, Pratylenchus crenatus, Pratylenchus neglectus and Pratylenchus penetrans) have worldwide distribution, especially in the temperate zones (Rivoal \& Cook, 1993). Nicol et al. (2002) reported that both $P$. thornei and $P$. neglectus can occur individually or mixed in soil of Turkish wheat growing regions.

RLN have a wide host range and can be difficult to identify and control. Symptoms on plants caused by RLN are non-specific and easily confused with stress from nutrient deficiency, drought and/or disease. The use of host-plant resistance is one of the most effective methods of controlling RLN. Resistance is defined as the ability of the host to inhibit nematode multiplication in plant (Rivoal \& Cook, 1993). It can range from low to moderate (partial or intermediate) resistance, to high resistance. Preferably, resistant germplasm should be combined with tolerance, which is the ability of the host plant to maintain high yield potential in the presence of the nematode (Rivoal \& Cook, 1993). The use of resistant varieties is an effective option to control RLN and it also environmentally sound, has no cost and requires no additional equipment. So far, there are no commercial wheat varieties with complete resistance to RLN ( $P$. thornei and $P$. neglectus). But, there are some alternative sources of partial resistance to RLN which have been identified in wild forms of wheat (Thompson \& Haak, 1997; Sheedy \& Thompson, 2009; Toktay et al., 2012a).

The best strategy for wheat improvement against diseases and pest is to the use of the valuable genetic resources of its wild progenitors (Peng et al., 2011). Emmer wheat (Triticum dicoccoides) is one of the earliest domesticated plants and has been a staple crop over millennia (Zahireva et al., 2010; Ozkan et al., 2002). T. dicoccoides, occurs naturally across the Fertile Crescent (Peng et al., 2011) and is a rich genetic resource to improve resistance to diseases and pest for breeding programs.

The objectives of this study were to screen wild emmer wheat and some national spring wheat varieties to find new sources of resistance to both $P$. thornei and $P$. neglectus and to identify varieties with superior resistance to current wheat varieties.

\section{Material and Methods}

In this study, 42 Turkish spring wheat varieties and 32 wild Emmer accessions as well as reference cultivars (check lines) were screened for resistance to $P$. thornei and $P$. neglectus under controlled growth room conditions.

The wild Emmers were collected from South-eastern Anatolia during 2004 and 2006 and were maintained in a wheat nursery collection by the Department of Crop Science in Cukurova University.

The wheat cultivars Gatcher and Seri were used as susceptible control and (CROC_1/AE.SQUARROSA (224)//OPATA) was used as a moderately resistant control against both $P$. thornei and $P$. neglectus (Sheedy \& Thompson, 2009). The Australian wheat cultivar GS50a was used as a moderately resistant control for $P$. thornei and a susceptible control for $P$. neglectus (Thompson, 2008; Sheedy et al., 2012). 


\section{Experimental procedures}

Seeds were surface sterilized with $1 \%$ sodium hypochlorite solution for 1 min and then rinsed 3 times with sterilized distilled water. To enhance seed germination, about 20 seeds of each accession were placed in $9 \mathrm{~cm}$ diameter petri dishes. These petri dishes were provided with a filter paper, which was moistened with sterile water and kept incubated at $20^{\circ} \mathrm{C}$ for 48 hours. After then, semi-rooted plants with root length about $3 \mathrm{~cm}$ were transplanted into the tubes $(3 \mathrm{~cm}$ diam. $\times 13 \mathrm{~cm}$ in long) filled with mixture of sand and soil $(9: 1 \mathrm{v} / \mathrm{v})$. Plants were left to grow in a growth room at $23^{\circ} \mathrm{C}$ and 16 hours of artificial supplementary light (Toktay et al., 2012b).

Plants were harvested after 9 weeks of nematodes inoculation. Final numbers of nematodes were extracted from both soil and roots using a Baermann funnel technique and kept in a misting chamber for 6 days to facilitate extraction. The nematodes were counted to determine resistant reaction of the different germplasm and compared them to known check lines. Each plant was replicated 7 times placed in a randomized block design in this experiment.

\section{Nematode inoculum culture and inoculation procedure}

Nematodes were grown in-vitro on carrot cultures according to Moody et al. (1973). The nematodes were extracted from the carrot culture by chopping the carrot disks and placing them into a misting chamber for 2-4 days. Nematodes were collected and amended to the required concentration per one $\mathrm{ml}$ of water. $P$. thornei and $P$. neglectus individuals collected from Southeastern part of Turkey, were counted under a binocular microscope and suspended tap water in $50 \mathrm{ml}$ flasks. Then one week of sowing, each plant was injected with $1 \mathrm{ml}$ of water consisting of 400 individuals in $1 \mathrm{ml}$ water. Plants were planted in growth room at $23-25^{\circ} \mathrm{C}$ temperature and during 16 hours of illumination for 9 weeks (Toktay et al., 2012b).

\section{Nematode extraction from plant roots}

After 9 weeks, plant shoots were removed and the nematodes were extracted from the soil and the roots using a Baermann funnel kept in a misting chamber for 6 days to extract the nematodes. One millilitre of nematode suspension was counted with three replications in a counting slide under a stereomicroscope at 32-fold magnification. The number of extracted nematodes per plant was calculated.

\section{Statistical design and analysis}

The number of nematodes per plant is an appropriate estimate of plant resistance (Keil et al., 2009; Sheedy et al., 2009; Farsi et al., 1995; Toktay et al., 2012b). The Reproduction factor (Rf) generally used in resistance experiments in nematology were used to give quantitative value of resistance (Keil et al., 2009). In the screening experiments under the laboratory conditions, nematode initial population $(\mathrm{Pi})$ set as inoculum level and nematode final population ( $\mathrm{Pf}$ ) and $\mathrm{Pf} / \mathrm{Pi}$ which defined as the reproduction factor of nematodes should be carefully emphasise to identify resistant reaction of the germplasm (Toktay et al., 2012b).

Wheat varieties were classified as completely resistant $(R)$, if no nematode multiplication in plant roots and soil was observed. Moderately resistant if nematode reproduction factor was under $1(R F<1)$, and finally if the $\mathrm{RF}$ is higher than 1 this line considers as susceptible. Number of nematodes were compared also according to susceptible and resistance check lines.

The total number of RLN's both root and soil in the pot for each plant were counted under microscope. Resistance was evaluated from the reproduction factor calculated by dividing the final by the initial $P$. thornei numbers in soil plus roots (Sheedy et al., 2009; Keil et al., 2009). Data were also analyzed by ANOVA and means were separated using Duncan's Multiple Range Test at $P<0.05$ significance level. All statistical analyses were performed by SPSS 16.0 (SPSS Inc. Illinois, USA). 


\section{Results and Discussion}

None of the screened wheat germplasm (15 bread wheat, 27 durum wheat and 32 emmer wheat genotypes collected from southeast part of Turkey) has completely resistance reaction against $P$. thornei and $P$. neglectus (Table 1, 2). Four check lines were used in the experiment ( 2 susceptible and 2 resistant) for $P$. thornei. Eleven durum wheat cultivars and six emmer lines were moderately resistant to the two root lesion nematodes. Four bread, twelve durum and nine emmers were moderately resistant against $P$. thornei (Table 1).

Table 1. The resistance reaction of Turkish wheat varieties and wild emmers with resistant and susceptible check lines against Turkish population of Pratylenchus thornei

\begin{tabular}{|c|c|c|c|c|c|c|}
\hline $\begin{array}{c}\text { Accession } \\
\text { no }\end{array}$ & Genotypes & $\begin{array}{l}\text { Wheat } \\
\text { type }\end{array}$ & $\begin{array}{c}\mathrm{Pi} \text { (Initial P. thornei } \\
\text { population) }\end{array}$ & $\begin{array}{l}\text { Pf (Final nematod } \\
\text { Population) } \pm \text { SE }\end{array}$ & $\begin{array}{c}\text { Rf (Reproduction } \\
\text { factor) }\end{array}$ & Reaction $^{*}$ \\
\hline 1 & *Gatcher & Bread & 400 & $399,29 \pm 3,62$ & 1,00 & $S$ \\
\hline 2 & ${ }^{*}$ Croc. & Bread & 400 & $205,14 \pm 3,14$ & 0,51 & MR \\
\hline 3 & *Serı & Bread & 400 & $564,86 \pm 2,77$ & 1,41 & $S$ \\
\hline 4 & ${ }^{*}$ GS50a & Bread & 400 & $117,57 \pm 4,88$ & 0,29 & MR \\
\hline 12 & Adana 99 & Bread & 400 & $213,86 \pm 2,89$ & 0,53 & MR \\
\hline 9 & Doğankent-1 & Bread & 400 & $378,00 \pm 4,63$ & 0,95 & MR \\
\hline 17 & Ceyhan-99 & Bread & 400 & $380,29 \pm 3,43$ & 0,95 & MR \\
\hline 10 & Karatopak & Bread & 400 & $383,57 \pm 3,4$ & 0,96 & MR \\
\hline 14 & Pandas & Bread & 400 & $390,29 \pm 3,16$ & 0,98 & $S$ \\
\hline 18 & Yüregir-89 & Bread & 400 & $412,43 \pm 3,87$ & 1,03 & $S$ \\
\hline 6 & Karakılçık & Bread & 400 & $475,29 \pm 5,61$ & 1,19 & S \\
\hline 8 & Cemre & Bread & 400 & $478,00 \pm 4,77$ & 1,20 & $S$ \\
\hline 15 & Çukurova-86 & Bread & 400 & $483,57 \pm 3,73$ & 1,21 & $S$ \\
\hline 13 & Seri-82 & Bread & 400 & $499,71 \pm 4,11$ & 1,25 & $S$ \\
\hline 5 & Karacadağ 98 & Bread & 400 & $501,57 \pm 5,68$ & 1,25 & $S$ \\
\hline 11 & Seyhan 95 & Bread & 400 & $505,29 \pm 4,23$ & 1,26 & $S$ \\
\hline 16 & Osmaniyem & Bread & 400 & $518,71 \pm 2,28$ & 1,30 & $S$ \\
\hline 7 & Nurkent & Bread & 400 & $532,43 \pm 8,73$ & 1,33 & $S$ \\
\hline 40 & Sogol Acırlı & Durum & 400 & $207,14 \pm 3,21$ & 0,52 & MR \\
\hline 19 & Fuatbey 208 & Durum & 400 & $209,43 \pm 3,72$ & 0,52 & MR \\
\hline 39 & Sarı bursa & Durum & 400 & $218,00 \pm 3,32$ & 0,55 & MR \\
\hline 44 & Minoret & Durum & 400 & $218,57 \pm 2,52$ & 0,55 & MR \\
\hline 36 & Siverek & Durum & 400 & $221,29 \pm 2,35$ & 0,55 & MR \\
\hline 27 & Hacıhalil & Durum & 400 & $268,57 \pm 5,52$ & 0,67 & MR \\
\hline 21 & Amanos-97 & Durum & 400 & $300,71 \pm 4,33$ & 0,75 & MR \\
\hline 31 & Zenit & Durum & 400 & $313,86 \pm 3,17$ & 0,78 & MR \\
\hline 41 & İskenderi & Durum & 400 & $349,71 \pm 13,9$ & 0,87 & MR \\
\hline 37 & Şırnak & Durum & 400 & $373,00 \pm 4,06$ & 0,93 & MR \\
\hline 26 & Bagacak96m & Durum & 400 & $376,43 \pm 3,18$ & 0,94 & MR \\
\hline 33 & Giberunda & Durum & 400 & $385,57 \pm 3,43$ & 0,96 & MR \\
\hline 30 & Dicle 74-M & Durum & 400 & $396,71 \pm 2,67$ & 0,99 & $S$ \\
\hline 23 & Menceki-2 & Durum & 400 & $398,57 \pm 3,54$ & 1,00 & $S$ \\
\hline 38 & Menceki-M & Durum & 400 & $405,86 \pm 4,36$ & 1,01 & $S$ \\
\hline 20 & Gediz.75 & Durum & 400 & $412,43 \pm 4,24$ & 1,03 & $S$ \\
\hline
\end{tabular}

${ }^{*} \mathrm{RF}<1=\mathrm{R}, 0,5<\mathrm{RF}<1=\mathrm{MR}, \mathrm{RF}>1=\mathrm{S}$ 
Table 1. The resistance reaction of Turkish wheat varieties and wild emmers with resistant and susceptible check lines against Turkish population of Pratylenchus thornei (continued)

\begin{tabular}{|c|c|c|c|c|c|c|}
\hline $\begin{array}{l}\text { Accession } \\
\text { no }\end{array}$ & Genotypes & $\begin{array}{l}\text { Wheat } \\
\text { type }\end{array}$ & $\begin{array}{l}\text { Pi (Initial } P . \text { thornei } \\
\text { population) }\end{array}$ & $\begin{array}{l}\text { Pf (Final nematod } \\
\text { Population) } \pm \text { SE }\end{array}$ & $\begin{array}{l}\text { Rf (Reproduction } \\
\text { factor) }\end{array}$ & Reaction* \\
\hline 42 & Kurtalan & Durum & 400 & $415,86 \pm 2,2$ & 1,04 & $S$ \\
\hline 32 & Şiraslan & Durum & 400 & $417,29 \pm 2,9$ & 1,04 & $S$ \\
\hline 46 & Şırnak Akkaya & Durum & 400 & $419,14 \pm 2,96$ & 1,05 & $S$ \\
\hline 28 & Akbugday & Durum & 400 & $419,29 \pm 3,69$ & 1,05 & $S$ \\
\hline 22 & Sham-1 & Durum & 400 & $442,57 \pm 3,28$ & 1,11 & $S$ \\
\hline 43 & Sorgül & Durum & 400 & $442,57 \pm 2,25$ & 1,11 & $S$ \\
\hline 35 & Beyaziyem & Durum & 400 & $474,29 \pm 2,93$ & 1,19 & $S$ \\
\hline 45 & Sorgül-2 & Durum & 400 & $474,57 \pm 3,32$ & 1,19 & $S$ \\
\hline 25 & Selçuklu & Durum & 400 & $481,86 \pm 4,02$ & 1,20 & $S$ \\
\hline 29 & Havrani & Durum & 400 & $504,86 \pm 3,28$ & 1,26 & $S$ \\
\hline 34 & A-97 & Durum & 400 & $517,29 \pm 3,39$ & 1,29 & $S$ \\
\hline 24 & Hav-27 & Durum & 400 & $517,43 \pm 2,62$ & 1,29 & $S$ \\
\hline 73 & Triticum.dicoccoides & Emmer & 400 & $198,29 \pm 9,4$ & 0,50 & MR \\
\hline 57 & T. dicoccoides & Emmer & 400 & $204,29 \pm 8,52$ & 0,51 & MR \\
\hline 64 & T. dicoccoides & Emmer & 400 & $205,14 \pm 8,61$ & 0,51 & MR \\
\hline 70 & T. dicoccoides & Emmer & 400 & $211,86 \pm 10,39$ & 0,53 & MR \\
\hline 51 & T. dicoccoides & Emmer & 400 & $237,29 \pm 13,13$ & 0,59 & MR \\
\hline 58 & T. dicoccoides & Emmer & 400 & $280,86 \pm 22,89$ & 0,70 & MR \\
\hline 63 & T. dicoccoides & Emmer & 400 & $332,71 \pm 14,14$ & 0,83 & MR \\
\hline 67 & T. dicoccoides & Emmer & 400 & $333,29 \pm 7,68$ & 0,83 & MR \\
\hline 48 & T. dicoccoides & Emmer & 400 & $333,43 \pm 13,49$ & 0,83 & MR \\
\hline 74 & T. dicoccoides & Emmer & 400 & $389,57 \pm 10,13$ & 0,97 & $S$ \\
\hline 61 & T. dicoccoides & Emmer & 400 & $430,14 \pm 13,72$ & 1,08 & $S$ \\
\hline 52 & T. dicoccoides & Emmer & 400 & $440,43 \pm 12,19$ & 1,10 & $S$ \\
\hline 66 & T. dicoccoides & Emmer & 400 & $444,29 \pm 12,05$ & 1,11 & $S$ \\
\hline 71 & T. dicoccoides & Emmer & 400 & $449,43 \pm 12,36$ & 1,12 & $S$ \\
\hline 78 & T. dicoccoides & Emmer & 400 & $450,57 \pm 11,29$ & 1,13 & $S$ \\
\hline 75 & T. dicoccoides & Emmer & 400 & $451,29 \pm 18,14$ & 1,13 & $S$ \\
\hline 55 & T. dicoccoides & Emmer & 400 & $453,00 \pm 11,69$ & 1,13 & $S$ \\
\hline 47 & T. dicoccoides & Emmer & 400 & $466,00 \pm 12,51$ & 1,17 & $S$ \\
\hline 72 & T. dicoccoides & Emmer & 400 & $494,00 \pm 11,74$ & 1,24 & $S$ \\
\hline$m$ & T d:D...... & $r-\ldots$ & $1 n n$ & $\operatorname{EnO} 71,1[11$ & 1 1 & $n$ \\
\hline 60 & T. dicoccoides & Emmer & 400 & $525,86 \pm 12,47$ & 1,31 & $\mathrm{~S}$ \\
\hline 50 & T. dicoccoides & Emmer & 400 & $527,29 \pm 10,15$ & 1,32 & $S$ \\
\hline 53 & T. dicoccoides & Emmer & 400 & $527,29 \pm 14,5$ & 1,32 & $S$ \\
\hline 56 & T. dicoccoides & Emmer & 400 & $527,43 \pm 14,13$ & 1,32 & $S$ \\
\hline 62 & T. dicoccoides & Emmer & 400 & $529,14 \pm 11,72$ & 1,32 & $S$ \\
\hline 77 & T. dicoccoides & Emmer & 400 & $531,14 \pm 14,46$ & 1,33 & $S$ \\
\hline 59 & T. dicoccoides & Emmer & 400 & $544,86 \pm 13,93$ & 1,36 & $S$ \\
\hline 65 & T. dicoccoides & Emmer & 400 & $546,00 \pm 15,75$ & 1,37 & $S$ \\
\hline 54 & T. dicoccoides & Emmer & 400 & $550,43 \pm 13,9$ & 1,38 & $S$ \\
\hline 49 & T. dicoccoides & Emmer & 400 & $558,29 \pm 20,4$ & 1,40 & $S$ \\
\hline 76 & T. dicoccoides & Emmer & 400 & $571,29 \pm 13,91$ & 1,43 & $S$ \\
\hline 68 & T. dicoccoides & Emmer & 400 & $584,57 \pm 14,17$ & 1,46 & $S$ \\
\hline
\end{tabular}

${ }^{*} \mathrm{RF}<1=\mathrm{R}, 0,5<\mathrm{RF}<1=\mathrm{MR}, \mathrm{RF}>1=\mathrm{S}$ 
There is one cultivar was as resistant control in the experiment against $P$. neglectus. GS50A was resistant to $P$. thornei, whereas it was susceptible against $P$. neglectus.

In this experiment, only two bread wheat, twenty durum wheat and thirteen emmers of wheat were found moderately resistant against $P$. neglectus (Table 2).

Table 2. The resistance reaction of Turkish wheat varieties and wild emmers with resistant and susceptible check lines against Turkish population of Pratylenchus neglectus

\begin{tabular}{|c|c|c|c|c|c|c|}
\hline $\begin{array}{c}\text { Accession } \\
\text { no }\end{array}$ & Genotypes & $\begin{array}{c}\text { Wheat } \\
\text { type }\end{array}$ & $\begin{array}{c}\mathrm{Pi} \text { (Initial } P \text {. neglectus } \\
\text { population) }\end{array}$ & $\begin{array}{c}\text { Pf (Final nematod } \\
\text { Population) } \pm \text { SE }\end{array}$ & $\begin{array}{c}\text { Rf (Reproduction } \\
\text { factor }\end{array}$ & Reaction $^{*}$ \\
\hline 1 & ${ }^{*}$ Gatcher & Bread & 400 & $402,71 \pm 5,62$ & 1,01 & $S$ \\
\hline 2 & ${ }^{*}$ Croc. & Bread & 400 & $196,71 \pm 3,73$ & 0,49 & MR \\
\hline 3 & ${ }^{*}$ Serı & Bread & 400 & $483,29 \pm 4,63$ & 1,21 & $S$ \\
\hline 4 & ${ }^{*}$ GS50a & Bread & 400 & $477,43 \pm 3,64$ & 0,69 & $S$ \\
\hline 15 & Çukurova-86 & Bread & 400 & $376,29 \pm 3,26$ & 0,94 & MR \\
\hline 18 & Yüregir-89 & Bread & 400 & $377,43 \pm 4,85$ & 0,94 & MR \\
\hline 6 & Karakılçık & Bread & 400 & $394,14 \pm 3,32$ & 0,99 & $S$ \\
\hline 11 & Seyhan 95 & Bread & 400 & $399,86 \pm 4,01$ & 1,00 & $S$ \\
\hline 5 & Karacadağ 98 & Bread & 400 & $405,29 \pm 4,37$ & 1,01 & $S$ \\
\hline 17 & Ceyhan-99 & Bread & 400 & $414,43 \pm 3,61$ & 1,04 & $S$ \\
\hline 12 & Adana 99 & Bread & 400 & $416,57 \pm 2,60$ & 1,04 & $S$ \\
\hline 14 & Pandas & Bread & 400 & $440,57 \pm 3,94$ & 1,10 & $S$ \\
\hline 10 & Karatopak & Bread & 400 & $454,57 \pm 3,37$ & 1,14 & S \\
\hline 9 & Doğankent-1 & Bread & 400 & $471,57 \pm 3,38$ & 1,18 & $S$ \\
\hline 16 & Osmaniyem & Bread & 400 & $471,57 \pm 3,80$ & 1,18 & $S$ \\
\hline 8 & Cemre & Bread & 400 & $502,71 \pm 5,02$ & 1,26 & $S$ \\
\hline 7 & Nurkent & Bread & 400 & $505,29 \pm 3,70$ & 1,26 & $S$ \\
\hline 13 & Seri-82 & Bread & 400 & $506,86 \pm 3,72$ & 1,27 & $S$ \\
\hline 41 & İskenderi & Durum & 400 & $197,71 \pm 3,23$ & 0,49 & MR \\
\hline 43 & Sorgül & Durum & 400 & $206,71 \pm 3,56$ & 0,52 & MR \\
\hline 24 & Hav-27 & Durum & 400 & $208,14 \pm 3,28$ & 0,52 & MR \\
\hline 31 & Zenit & Durum & 400 & $213,71 \pm 3,95$ & 0,53 & MR \\
\hline 37 & Şırnak & Durum & 400 & $217,71 \pm 2,23$ & 0,54 & MR \\
\hline 26 & Bagacak96m & Durum & 400 & $249,57 \pm 3,08$ & 0,62 & MR \\
\hline 30 & Dicle 74-M & Durum & 400 & $250,57 \pm 3,26$ & 0,63 & MR \\
\hline 27 & Hacıhalil & Durum & 400 & $273,43 \pm 3,00$ & 0,68 & MR \\
\hline 33 & Giberunda & Durum & 400 & $286,71 \pm 3,28$ & 0,72 & MR \\
\hline 29 & Havrani & Durum & 400 & $305,86 \pm 3,79$ & 0,76 & MR \\
\hline 19 & Fuatbey 208 & Durum & 400 & $307,00 \pm 4,04$ & 0,77 & MR \\
\hline 39 & Sarı bursa & Durum & 400 & $307,14 \pm 6,15$ & 0,77 & MR \\
\hline 20 & Gediz.75 & Durum & 400 & $313,57 \pm 4,38$ & 0,78 & MR \\
\hline 32 & Şiraslan & Durum & 400 & $315,71 \pm 2,75$ & 0,79 & MR \\
\hline 40 & Sogol Acırlı & Durum & 400 & $338,14 \pm 3,61$ & 0,85 & MR \\
\hline 21 & Amanos-97 & Durum & 400 & $339,29 \pm 3,22$ & 0,85 & MR \\
\hline 42 & Kurtalan & Durum & 400 & $341,86 \pm 3,32$ & 0,85 & MR \\
\hline 23 & Menceki-2 & Durum & 400 & $346,57 \pm 2,69$ & 0,87 & MR \\
\hline 38 & Menceki-M & Durum & 400 & $378,71 \pm 3,34$ & 0,95 & MR \\
\hline 44 & Minoret & Durum & 400 & $380,71 \pm 2,83$ & 0,95 & MR \\
\hline 35 & Beyaziyem & Durum & 400 & $398,29 \pm 2,88$ & 1,00 & $S$ \\
\hline 46 & Şırnak Akkaya & Durum & 400 & $394,43 \pm 3,52$ & 0,99 & $S$ \\
\hline 28 & Akbugday & Durum & 400 & $401,00 \pm 15,49$ & 1,00 & $S$ \\
\hline 36 & Siverek & Durum & 400 & $415,86 \pm 2,34$ & 1,04 & $S$ \\
\hline 34 & A-97 & Durum & 400 & $441,71 \pm 3,34$ & 1,10 & $S$ \\
\hline 45 & Sorgül-2 & Durum & 400 & $469,14 \pm 3,97$ & 1,17 & S \\
\hline
\end{tabular}

\footnotetext{
${ }^{*} \mathrm{RF}<1=\mathrm{R}, 0,5<\mathrm{RF}<1=\mathrm{MR}, \mathrm{RF}>1=\mathrm{S}$
} 
Table 2. The resistance reaction of Turkish wheat varieties and wild emmers with resistant and susceptible check lines against Turkish population of Pratylenchus neglectus (continued)

\begin{tabular}{|c|c|c|c|c|c|c|}
\hline $\begin{array}{c}\text { Accession } \\
\text { no }\end{array}$ & Genotypes & $\begin{array}{l}\text { Wheat } \\
\text { type }\end{array}$ & $\begin{array}{c}\mathrm{Pi} \text { (İnitial P. neglectus } \\
\text { population) }\end{array}$ & $\begin{array}{l}\text { Pf (Final nematod } \\
\text { Population) } \pm \text { SE }\end{array}$ & $\begin{array}{c}\text { Rf (Reproduction } \\
\text { factor }\end{array}$ & Reaction* \\
\hline 22 & Sham-1 & Durum & 400 & $473,71 \pm 2,69$ & 1,18 & $S$ \\
\hline 25 & Selçuklu & Durum & 400 & $492,00 \pm 3,70$ & 1,23 & S \\
\hline 57 & Triticum.dicoccoi & Emmer & 400 & $193,71 \pm 15,98$ & 0,48 & MR \\
\hline 67 & T. dicoccoides & Emmer & 400 & $194,29 \pm 11,67$ & 0,49 & MR \\
\hline 64 & T. dicoccoides & Emmer & 400 & $201,14 \pm 7,56$ & 0,50 & MR \\
\hline 73 & T. dicoccoides & Emmer & 400 & $206,86 \pm 12,54$ & 0,52 & MR \\
\hline 77 & T. dicoccoides & Emmer & 400 & $294,71 \pm 16,66$ & 0,74 & MR \\
\hline 62 & T. dicoccoides & Emmer & 400 & $314,43 \pm 17,22$ & 0,79 & MR \\
\hline 61 & T. dicoccoides & Emmer & 400 & $328,14 \pm 12,97$ & 0,82 & MR \\
\hline 58 & T. dicoccoides & Emmer & 400 & $330,29 \pm 14,70$ & 0,83 & MR \\
\hline 54 & T. dicoccoides & Emmer & 400 & $332,71 \pm 17,80$ & 0,83 & MR \\
\hline 74 & T. dicoccoides & Emmer & 400 & $356,71 \pm 13,97$ & 0,89 & MR \\
\hline 66 & T. dicoccoides & Emmer & 400 & $358,57 \pm 16,04$ & 0,90 & MR \\
\hline 47 & T. dicoccoides & Emmer & 400 & $373,71 \pm 13,38$ & 0,93 & MR \\
\hline 70 & T. dicoccoides & Emmer & 400 & $391,00 \pm 15,90$ & 0,98 & MR \\
\hline 53 & T. dicoccoides & Emmer & 400 & $427,57 \pm 12,18$ & 1,07 & $\mathrm{~s}$ \\
\hline 78 & T. dicoccoides & Emmer & 400 & $433,14 \pm 12,87$ & 1,08 & s \\
\hline 75 & T. dicoccoides & Emmer & 400 & $436,14 \pm 12,28$ & 1,09 & S \\
\hline 65 & T. dicoccoides & Emmer & 400 & $441,86 \pm 15,06$ & 1,10 & S \\
\hline 48 & T. dicoccoides & Emmer & 400 & $447,29 \pm 15,05$ & 1,12 & s \\
\hline 72 & T. dicoccoides & Emmer & 400 & $449,43 \pm 14,83$ & 1,12 & S \\
\hline 59 & T. dicoccoides & Emmer & 400 & $452,43 \pm 12,22$ & 1,13 & S \\
\hline 51 & T. dicoccoides & Emmer & 400 & $453,00 \pm 16,53$ & 1,13 & s \\
\hline 50 & T. dicoccoides & Emmer & 400 & $480,00 \pm 22,81$ & 1,20 & s \\
\hline 55 & T. dicoccoides & Emmer & 400 & $481,29 \pm 11,03$ & 1,20 & S \\
\hline 68 & T. dicoccoides & Emmer & 400 & $526,29 \pm 11,17$ & 1,32 & s \\
\hline 69 & T. dicoccoides & Emmer & 400 & $528,71 \pm 19,45$ & 1,32 & S \\
\hline 49 & T. dicoccoides & Emmer & 400 & $529,14 \pm 10,05$ & 1,32 & s \\
\hline 60 & T. dicoccoides & Emmer & 400 & $531,71 \pm 13,87$ & 1,33 & S \\
\hline 63 & T. dicoccoides & Emmer & 400 & $533,86 \pm 12,72$ & 1,33 & S \\
\hline 76 & T. dicoccoides & Emmer & 400 & $536,71 \pm 15,14$ & 1,34 & S \\
\hline 56 & T. dicoccoides & Emmer & 400 & $538,43 \pm 11,37$ & 1,35 & S \\
\hline 71 & T. dicoccoides & Emmer & 400 & $538,71 \pm 15,55$ & 1,35 & S \\
\hline 52 & T. dicoccoides & Emmer & 400 & $539,14 \pm 15,82$ & 1,35 & s \\
\hline
\end{tabular}

${ }^{*} \mathrm{RF}<1=\mathrm{R}, 0,5<\mathrm{RF}<1=\mathrm{MR}, \mathrm{RF}>1=\mathrm{S}$

Using resistant varieties to control the root lesion nematodes is the main target of many nematologists and breeders around all over the world due to its adaptability and easy to be applied once identified. There are very limited researches to determine resistance of local cultivars to root lesion nematodes in Turkey. Toktay et al. (2008) found resistant in some local varieties in East Mediterranean region of Turkey. Imren et al. (2013), reported that wheat cv. Adana 99 is moderately resistant to both cereal cyst nematode (Heterodera avenae) pathotype Ha 21 and root lesion nematode ( $P$. thornei).

Durable resistance has generally been transferred from wild relatives to cultivated crops (Boerma \& Hussey, 1992). The southeastern Anatolia region Karacadağ-Diyarbakir is rich in genetic resources of wild emmer, $T$. dicoccoides, T. urartu, and T. boeticum as important wheat wild relatives of wheat (Nesbit \& Samuel, 1998; Salamina et al., 2002; Ozkan et al., 2011). Therefore wild emmer of wheat has been obtained from the Southeastern Anatolia region to assess resistance reaction of RLN. 
There have been many studies to identify new sources of resistance to RLN on Middle Eastern wheat varieties and landraces (Nicol et al., 2005; Schimdt et al., 2005; Zwart et al., 2005; Thompson, 2008; Thompson \& Seymour 2011; Toktay et al., 2012a). One of the particular relevance to Australian wheat improvement programs were the $P$. thornei resistant accessions identified among Iranian landraces (Sheedy \& Thompson, 2009) and wild relatives of wheat including T. dicoccoides (Sheedy et al., 2012). In this study, most varieties and wild relatives from this region in Turkey were resistant; these results were in agreement with those of Toktay et al. (2012a). Farsi et al. (1995) showed that GS50a was susceptible to $P$. neglectus, indicating that resistance to $P$. thornei does not convey resistance to $P$. neglectus. Imren et al. (2013) found 17 emmer wheat and 4 local wheat varieties resistant against Ha 21 pathotype of $H$. avenae.

In Turkey, the soil borne diseases, especially plant parasitic nematodes, have not been given attention and are under exploited by breeders. Also, the Turkish farmers have been planting their local seeds without having any idea of nematodes and their potential to cause significant losses. Such ignorant and continued plantation of susceptible wheat cultivar is expected to accelerate the nematode population that will ultimately lead to huge amount of their grain yield losses. Both emmer and cultivated wheat collections germplasms are useful to understanding the genetic basis for resistance, determining the gene(s) responsible, and identifying which may be of great benefit to breeding programs by pyramiding different resistance genes into single lines.

In this this study, a total of 74 lines were evaluated for RLN resistance. Unfortunately, there were no cultivars with complete resistance to either RLN species. But, 17 durum wheat and four wild emmers were moderately resistant to both nematodes. These cultivars can be used to improve RLN resistance in commercial cultivars and to identify molecular markers for resistance to RLN through association mapping. More detailed experiments with local varieties and wild relatives are required to find new sources of resistance that can be used in both national and international wheat breeding programs.

\section{Acknowledgements}

This publication was supported by Tubitak Project number 1120 565. The authors thank Prof. Dr. Hakan Ozkan (University of Cukurova) for selecting and supplying the Emmer wheats and Turkish varieties used in the study.

\section{References}

TUIK, 2013. Turkish Statistical Institute. www.tuik.gov.tr (Accces date:14.02.2014).

Boerma, H. R. \& R. S. Hussey, 1992. Breeding plants for resistance to nematodes. Journal of Nematology, 24: 242252.

Imren, M., H. Toktay, R. Bozbuga, A. Dababat \& I. H. Elekçioğlu, 2013. Determination of resistance against to Cereal cyst nematode, Heterodera avenae (Wollenweber, 1924) in some wheat germplasm. Turkish Journal of Entomology, 37: 229-238.

Keil, T., E. Laubach, S. Sharma \& C. Jung, 2009. Screening for resistance in the primary and secondary gene pool of barley against the root-lesion nematode Pratylenchus neglectus. Plant Breeding, 128: 436-442.

Mc Donald, A. H. \& J. M. Nicol, 2005. "Nematode parasites of cereals, 131-192". In: Plant Parasitic Nematodes in Subtropical and Tropical Agriculture. (Eds: Luc, M., R. A. Sikora, \& J. Bridge ). CAB International, London, UK, 896 pp.

Nesbit, M. \& D. Samuel, 1998. Wheat domestication, archeobotanical evidence. Science, 279: 14-33.

Nicol, J. M., R. Rivoal, N. Bolat, H. Aktas, H. Braun, M. Mergoum, A. F. Yıldırım, A. Bagcı, I. H. Elekcioğlu \& A. Yahyaoui, 2002. Frequency and diversity of the cyst and lesion nematodes on wheat in the Turkish central Anatolian plateau. Nematology, 4: 383-443. 
Özkan, H., A. Brandolini, R. Schafer-Pregl \& F. Salamini, 2002. AFLP analysis of a collection of tetraploid wheats indicates the origin of emmer and hard wheat domestication in Southeast Turkey. Molecular Biology and Evolution, 19: 1797-1801.

Özkan, H., G. Willcox, A. Graner, F. Salamini \& B. Kilian, 2011. Geographic distribution and domestication of wild emmer wheat (Triticum dicoccoides). Genetic Resources and Crop Evolution, 58: 11-53.

Salamini, F., H. Özkan, A. Brandolin, R. Schafer-Pregl \& W. Martin, 2002. Genetics and geomorphology of wild cereal domestication in the near east. Nature Genetics, 3: 429-441.

Sheedy, J. G. \& J. P. Thompson, 2009. Resistance to the root-lesion nematode Pratylenchus thornei of Iranian landrace wheat. Australian Plant Pathology, 38: 478-489.

Thompson, J. P. \& M. I. Haak, 1997. Resistance to root-lesion nematode (Pratylenchus thornei) in Aegilops tauschii Coss., the D-genome donor to wheat. Australian Journal of Agricultural Research 48: 553-559.

Thompson, J. P. \& N. P. Seymour, 2011. Inheritance of resistance to root lesion nematode (Pratylenchus thornei) in wheat landraces and cultivars from the West Asia and North Africa (WANA) region. Crop and Pasture Science, 62: 82-93.

Thompson, J. P., 2008. Resistance to root-lesion nematodes (Pratylenchus thornei and P. neglectus) in synthetic hexpaloid wheats and their durum and Aegilops tauschii parents. Australian Journal of Agricultural Research, 59: 432-446.

Thompson, J. P., K. J. Owen, G. R. Stirling \& M. J. Bell, 2008. Root-lesion nematodes (Pratylenchus thornei and $P$. neglectus): a review of recent progress in managing a significant pest of grain crops in northern Australia. Australian Plant Pathology, 37: 235-242.

Toktay, H., 2008. Resistance of Some Spring Wheat Against Pratylenchus thornei Sher Et Allen Tylenchida: Pratylenchidae. PhD Thesis, University of Cukurova, Institute of Nature of Science, Adana, Turkey, $117 \mathrm{pp}$

Toktay, H., E. Yavuzaslanoğlu, M. İmren, J. M. Nicol, I. H. Elekçioğlu \& A. Dababat, 2012a. Screening for resistance to Heterodera filipjevi and Pratylenchus thornei in sister lines of spring wheat. Turkish Journal of Entomology, 36: 455-461.

Toktay, H., M. İmren, J. M. Nicol, A. Dababat \& I. H. Elekçioğlu, 2012b. Improved methodology for resistance screening in spring wheat against the root lesion nematode, Pratylenchus thornei (Sher et Allen) (Tylenchida: Pratylenchidae). Turkish Journal of Entomology, 36: 533-540.

Toktay, H., L. Mclntyre, J. M. Nicol, H. Ozkan, \& I. H. Elekcioglu, 2006. Identification of common root lesion nematode (Pratylenchus thornei Sher et Allen) loci in bread wheat. Genome, 49: 1319-1323.

Zaharieva, M., N. G. Ayana, A. Al Hakimi, S. C. Misra \& P. Monneveux, 2010. Cultivated emmer wheat (Triticum dicoccon Schrank), an old crop with promising future: a review. Crop Evolution, 57: 937-962.

Zwart, R. S., J. P. Thompson \& I. D. Godwin, 2005. Identification of quantitative trait loci for resistance to two species of root-lesion nematode (Pratylenchus thornei and P. neglectus) in wheat. Australian Journal of Agricultural Research, 56: 345-352. 